\title{
A NOTE ON STABLE EQUIVALENCE AND NAKAYAMA ALGEBRAS
}

\author{
IDUN REITEN
}

\begin{abstract}
Two artin algebras $\Lambda$ and $\Lambda^{\prime}$ are said to be stably equivalent if the categories of finitely generated modules modulo projective for $\Lambda$ and $\Lambda^{\prime}$ are equivalent categories.

If $\Lambda^{\prime}$ is stably equivalent to a Nakayama (i.e. generalized uniserial) algebra $\Lambda$, we prove that $\Lambda$ and $\Lambda^{\prime}$ have the same number of nonprojective simple modules. And if $\Lambda$ and $\Lambda^{\prime}$ are stably equivalent indecomposable Nakayama algebras where each indecomposable projective module has length at least 3 , then $\Lambda$ and $\Lambda^{\prime}$ have the same admissible sequences.
\end{abstract}

Introduction. We recall that two artin algebras $\Lambda$ and $\Lambda^{\prime}$ are said to be stably equivalent if the categories $\bmod \Lambda$ and $\bmod \Lambda^{\prime}$ modulo projectives are

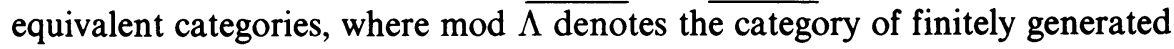
left $\Lambda$-modules.

An open problem about stable equivalence, which is of importance in the modular representation theory of finite groups, is whether two stably equivalent algebras $\Lambda$ and $\Lambda^{\prime}$ have the same number of nonprojective simple modules. In $\$ 1$ we show that if one of the algebras is a Nakayama (i.e. generalized uniserial) algebra, then this is the case. It is also known to be true if $\Lambda$ is hereditary [2, Theorem 5.5] and if $\Lambda$ has no nonzero projective injective modules [1, Proposition 3.6]. See also [11].

In $\S 2$ we prove the result, announced in [10], that if $\Lambda$ and $\Lambda^{\prime}$ are stably equivalent Nakayama algebras such that each indecomposable projective module has length at least 3 , then $\Lambda$ and $\Lambda^{\prime}$ have the same admissible sequences (see $\$ 2$ for definition). Hence if they are both basic algebras over an algebraically closed field, they must be isomorphic [8, Satz 7]. Our method is based upon using the notion of irreducible map introduced in [3].

In connection with the above, we point out that if $\Lambda^{\prime}$ is an artin algebra stably equivalent to a Nakayama algebra $\Lambda$, then $\Lambda^{\prime}$ is not necessarily Nakayama [9]. If, however, $\Lambda$ has Loewy length at most 3 , then $\Lambda^{\prime}$ is Nakayama [9, Corollary 2.2]. However, there are non Nakayama algebras $\Lambda^{\prime}$ stably equivalent to Nakayama algebras of Loewy length 4, and in [12] we give, as announced in [10], a characterization of the artin algebras stably equivalent to Nakayama algebras of Loewy length 4. P. Gabriel and C.

Received by the editors August 31, 1977 and, in revised form, January 3, 1978.

AMS (MOS) subject classifications (1970). Primary 16A46, 16A42; Secondary 16A64.

Key words and phrases. Nakayama algebra, stable equivalence. 
Riedtmann have studied artin algebras stably equivalent to self-injective algebras over an algebraically closed field. Stable equivalence of Nakayama algebras has also been studied in [6].

1. Let $\Lambda$ be an artin algebra, and denote by mod $\Lambda$ the category of finitely generated left $\Lambda$-modules. Denote by $\bmod \Lambda$ the category $\bmod \Lambda$ modulo projectives, that is, the objects of $\bmod \Lambda$ are the same as those of $\bmod \Lambda$, now denoted $\underline{M}$ for $M$ in $\bmod \Lambda$, and $\operatorname{Hom}(M, \underline{N})=\operatorname{Hom}_{\Lambda}(M, N) / P(M, N)$, where $P(M, N)$ consists of the maps from $M$ to $N$ which factor through a projective $\Lambda$-module. For $f: M \rightarrow N$, let $\underline{f}$ denote the image of $f$ in $\operatorname{Hom}(\underline{M}, \underline{N}) . \Lambda$ and $\Lambda^{\prime}$ are said to be stably equivalent if there is an equivalence of categories $\alpha: \bmod \Lambda \rightarrow \bmod \Lambda^{\prime} . \alpha$ induces a correspondence, also denoted by $\alpha$, between the objects in $\bmod _{P} \Lambda$ and in $\bmod _{P} \Lambda^{\prime}$, the categories of finitely generated modules with no nonzero projective summands.

The following lemmas about stable equivalence will be useful in what follows.

LemMa 1.1. Let $\Lambda$ be an artin algebra and $f: A \rightarrow B$ an epimorphism in $\bmod \Lambda$. If $A$ and $B$ have no nonzero projective summands, then $f$ is not zero.

Proof. If $\underline{f}$ was zero, we would have some commutative diagram

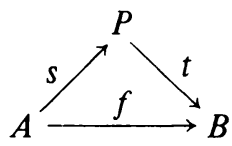

where $P$ is projective. Since $A$ has no nonzero projective summand $\operatorname{Im} s$ must be contained in $\mathrm{r} P$, where $\mathrm{r}$ denotes the radical of $\Lambda$. Hence $\operatorname{Im} f \subset \mathrm{r} B$, which contradicts the fact that $f$ is an epimorphism. It follows that $f$ is not zero.

LEMMA 1.2. Let $\Lambda$ be a Nakayama algebra and $f: A \rightarrow B$ a monomorphism. If $A$ and $B$ are indecomposable nonprojective $\Lambda$-modules, then $\underline{f}$ is not zero.

Proof. Assume that $\underline{f}$ is zero. We then have some commutative diagram

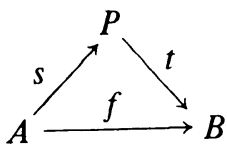

where $t: P \rightarrow B$ is a projective cover. Since $P$ is indecomposable, soc $P$ is simple, and since $t: P \rightarrow B$ is not a monomorphism, $t(\operatorname{soc} P$ ) must be zero. It follows that $f(\operatorname{soc} A)=t s(\operatorname{soc} A)$ is zero, since $s(\operatorname{soc} A) \subset \operatorname{soc} P$. This contradicts the fact that $f$ is a monomorphism. Hence it follows that $f$ is zero.

In this section we prove the following result, which under the assumption that $\Lambda$ and $\Lambda^{\prime}$ are self-injective, is essentially proved in [7].

THEOREM 1.3. Let $\Lambda$ and $\Lambda^{\prime}$ be stably equivalent artin algebras. If $\Lambda$ is 
Nakayama, then $\Lambda$ and $\Lambda^{\prime}$ have the same number of nonprojective simple modules.

Proof. Let $\alpha: \bmod \Lambda^{\prime} \rightarrow \bmod \Lambda$ be an equivalence, where $\Lambda$ is Nakayama. Let $S_{1}, \ldots, S_{n}$ be the nonprojective simple $\Lambda^{\prime}$-modules. We then claim that $\operatorname{soc}\left(\alpha S_{1}\right), \ldots, \operatorname{soc}\left(\alpha S_{n}\right)$ are nonisomorphic simple $\Lambda$-modules. Since $\Lambda$ is Nakayama, and the $\alpha S_{i}$ are all indecomposable, we know that the $\operatorname{soc}\left(\alpha S_{i}\right)$ are all simple. Assume that for $i \neq j, \operatorname{soc}\left(\alpha S_{i}\right) \cong \operatorname{soc}\left(\alpha S_{j}\right)$. We can assume without loss of generality that the length of $\alpha S_{i}$ is at most equal to the length of $\alpha S_{j}$. Since $\Lambda$ is Nakayama, we know by the structure of indecomposable $\Lambda$-modules that there is a monomorphism $t: \alpha S_{i} \rightarrow \alpha S_{j}$. Since $\operatorname{Hom}\left(S_{i}, S_{j}\right)=$ 0 , we have $\operatorname{Hom}\left(\alpha S_{i}, \alpha S_{j}\right)=0$, which is a contradiction by Lemma 1.2. This shows that the soc $\left(\alpha S_{i}\right)$ are all nonisomorphic.

We further observe that since $\operatorname{soc}\left(\alpha S_{i}\right)$ is a composition factor of some $P_{i} /$ soc $P_{i}$, where $P_{i}$ is indecomposable projective, $\operatorname{soc}\left(\alpha S_{i}\right)$ is not a simple projective $\Lambda$-module. Hence $\Lambda$ has at least $n$ nonisomorphic simple nonprojective modules.

Let now $T$ be a simple nonprojective $\Lambda$-module, and let $A$ be the indecomposable nonprojective $\Lambda^{\prime}$-module such that $\alpha A=T$. Then there is an epimorphism $t: A \rightarrow S_{i}$ for some $i$, hence we know by Lemma 1.1 that $\operatorname{Hom}\left(\underline{A}, S_{i}\right)$ is not zero. It then follows that $\operatorname{Hom}\left(\underline{T}, \alpha S_{i}\right)$ is not zero. This means that there is a nonzero map, hence a monomorphism, from $T$ to $\alpha S_{i}$, so that $T \cong \operatorname{soc} \alpha S_{i}$. This shows that $\Lambda$ has exactly $n$ simple nonprojective modules, and the proof is done.

2. Let $\Lambda$ be a Nakayama algebra. We shall recall some well known facts about indecomposable Nakayama algebras (see [8], [5]). There is an ordering of the indecomposable projective $\Lambda$-modules $P_{1}, \ldots, P_{n}$, such that $P_{i} / \mathrm{r} P_{i} \cong$ $\mathrm{r} P_{i+1} / \mathrm{r}^{2} P_{i+1}, i=1, \ldots, n-1$, and either $\mathrm{r} P_{n}$ is zero or $P_{n} / \mathrm{r} P_{n} \cong$ $\mathrm{r} P_{1} / \mathrm{r}^{2} P_{1}$. The sequence of integers $c_{1}, \ldots, c_{n}$, where $c_{i}$ is the length of $P_{i}$, is called an admissible sequence for $\Lambda$. An admissible sequence is determined up to cyclic permutation. If $\Lambda$ is a basic indecomposable Nakayama algebra over an algebraically closed field $k$, then the associated admissible sequence uniquely determines $\Lambda$ [8]. We recall that we showed in [9] that if $\Lambda$ and $\Lambda^{\prime}$ are stably equivalent indecomposable Nakayama algebras, with no simple projective modules then the associated admissible sequences have the same length, that is, the same number of $c_{i}$ (this also follows from Theorem 1.1, since it says that $\Lambda$ and $\Lambda^{\prime}$ have the same number of nonprojective simple modules), and that the number of $c_{i}$ equal to a given number $t \geqslant 2$ is the same for both admissible sequences. (See [9, Proposition 1.1].) In the special case that all $c_{i}$ are 3 or 4 , we showed that the admissible sequences are actually the same ([9, Proposition 3.1], [10, Proposition $\left.\left.3.1^{\prime}\right]\right)$. Here we shall, as announced in [10], prove the same thing just assuming that all $c_{i}$ are at least 3. We give an example to show that the result is not true in general.

To do this we shall use the notion of irreducible maps introduced in [3]. We recall that for an artin algebra $\Lambda$, a map $f: A \rightarrow B$ in $\bmod \Lambda$ is said to be 
irreducible if $f$ is neither a split epimorphism nor a split monomorphism, and given any commutative diagram

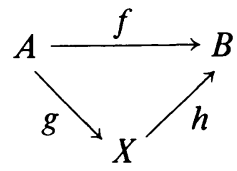

then either $g$ is a split monomorphism or $h$ is a split epimorphism. It is not hard to see that if $\Lambda$ is a Nakayama algebra, then a map $f: A \rightarrow B$ between indecomposable modules $A$ and $B$ in mod $\Lambda$ is irreducible if and only if $f$ is a monomorphism and $l(A)=l(B)-1$ or $f$ is an epimorphism and $l(A)=$ $l(B)+1$ (see [3]). Here $l$ denotes length. Another useful fact about irreducible maps that we shall need here is that if $\alpha: \bmod \Lambda \rightarrow \bmod \Lambda^{\prime}$ is an equivalence and $f: A \rightarrow B$ is an irreducible map where $A$ and $B$ are in $\bmod _{P} \Lambda$, then $f^{\prime}: A^{\prime} \rightarrow B^{\prime}$, where $\underline{A}^{\prime}=\alpha(A), \underline{B}^{\prime}=\alpha(B), \alpha(\underline{f})=\underline{f}^{\prime}$, is also irreducible [4, Proposition 12].

To prove that two stably equivalent indecomposable Nakayama algebras, where each indecomposable projective module has length at least 3 , have the same admissible sequences, we shall need a few lemmas.

LEMMA 2.1. Let $\Lambda$ be a Nakayama algebra.

(a) Let $S_{1}$ be a simple $\Lambda$-module and assume that the projective cover $P_{1}$ of $S_{1}$ has length at least 3. Then $S_{2}=\mathfrak{r} P_{1} / \mathfrak{r}^{2} P_{1}$ is the unique indecomposable nonprojective $\Lambda$-module with the property that there is some chain $S_{2} \stackrel{f}{\rightarrow} X \stackrel{g}{\rightarrow} S_{1}$ of irreducible maps between indecomposable nonprojective $\Lambda$-modules such that gf is zero.

(b) Let $P_{1}$ be an indecomposable projective $\Lambda$-module of length $n \geqslant 3$. Then there is a unique indecomposable nonprojective $\Lambda$-module $A$ such that there is some chain $A \stackrel{f}{\rightarrow} Y \stackrel{g}{\rightarrow} P_{1} / \mathfrak{r}^{n-1} P_{1}$ of irreducible maps between indecomposable nonprojective modules such that gf is zero, if and only if $\mathrm{r} P_{1}$ is not projective.

If $\mathrm{r} P_{1}$ is not projective, then $\bar{A} \cong \mathrm{r} P_{1}$.

Proof. (a) $P_{1} / \mathrm{r}^{2} P_{1}$ is the only indecomposable (nonprojective) $\Lambda$-module such that there is some irreducible map $P_{1} / \mathrm{r}^{2} P_{1} \rightarrow S_{1}$. The only indecomposable $\Lambda$-modules having irreducible maps to $P_{1} / \mathrm{r}^{2} P_{1}$ are $P_{1} / \mathrm{r}^{3} P_{1}$ and $\mathrm{r} P_{1} / \mathrm{r}^{2} P_{1}$ $=S_{2} . S_{2}$ is not projective, and for any chain

$$
S_{2} \stackrel{f}{\rightarrow} P_{1} / \mathrm{r}^{2} P_{1} \stackrel{g}{\rightarrow} S_{1}
$$

of irreducible maps, $g f$ is clearly zero. And if $P_{1} / \mathrm{r}^{3} P_{1}$ is not projective, and $P_{1} / \mathrm{r}^{3} P_{1} \stackrel{f}{\rightarrow} P_{1} / \mathrm{r}^{2} P_{1} \stackrel{g}{\rightarrow} S_{1}$ is a chain of irreducible maps, then $g f$ is an epimorphism, so that $\underline{g} \neq 0$ by Lemma 1.1 .

(b) $\mathfrak{r} P_{1} / \mathfrak{r}^{n-1} P_{1}$ is the only indecomposable nonprojective $\Lambda$-module with an irreducible map to $P_{1} / \mathfrak{r}^{n-1} P_{1}$. The indecomposable nonprojective modules with an irreducible map to $\mathrm{r} P_{1} / \mathrm{r}^{n-1} P_{1}$ are $\mathrm{r}^{2} P_{1} / \mathrm{r}^{n-1} P_{1}$, if $n \geqslant 4$, and $\mathrm{r} P_{1}$, if $\mathrm{r} P_{1}$ is not projective. For $n \geqslant 4$, any composition of irreducible maps 


$$
\mathrm{r}^{2} P_{1} / \mathrm{r}^{n-1} P_{1} \stackrel{f}{\rightarrow} \mathrm{r} P_{1} / \mathrm{r}^{n-1} P_{1} \stackrel{g}{\rightarrow} P_{1} / \mathrm{r}^{n-1} P_{1}
$$

is a monomorphism. Hence $g f$ is not zero by Lemma 1.2. If $\mathrm{r} P_{1}$ is not projective, let $\mathrm{r} P_{1} \stackrel{f}{\rightarrow} \mathrm{r} P_{1} / \mathrm{r}^{n-1} \stackrel{g}{P_{1}} \stackrel{g}{\rightarrow} P_{1} / \mathrm{r}^{n-1} P_{1}$ be a chain of irreducible maps between indecomposable nonprojective $\Lambda$-modules. It is then easy to see that $\underline{g f}=0$. This finishes the proof of $(\mathrm{b})$.

LEMMA 2.2. Let $\alpha: \bmod \Lambda \rightarrow \bmod \Lambda^{\prime}$ be a stable equivalence between

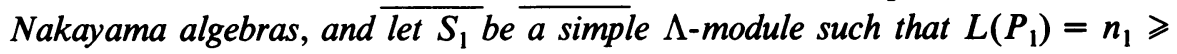
3 , where $P_{1}$ is a projective cover for $S_{1}$. Assume that $\alpha S_{1} \cong T_{1}$, where $T_{1}$ is a simple $\Lambda^{\prime}$-module, and denote by $Q_{1}$ a projective cover of $T_{1}$. Then $\alpha S_{2} \cong T_{2}$, where $S_{2}=\mathrm{r} P_{1} / \mathrm{r}^{2} P_{1}$ and $T_{2}=\mathrm{r} Q_{1} / \mathrm{r}^{2} Q_{1}$.

Proof. We have the following chains of irreducible maps between indecomposable nonprojective $\Lambda^{\prime}$-modules.

$$
\alpha S_{2} \stackrel{f}{\rightarrow} \alpha\left(P_{1} / \mathrm{r}^{2} P_{1}\right) \stackrel{g}{\rightarrow} \alpha S_{1}=T_{1} \quad \text { and } \quad T_{2} \stackrel{f^{\prime}}{\rightarrow} Q_{1} / \mathrm{r}^{2} Q_{1} \stackrel{g^{\prime}}{\rightarrow} T_{1}
$$

To get the first chain, we first consider $S_{2} \rightarrow P_{1} / \mathrm{r}^{2} P_{1} \rightarrow S_{1}$, and use the connection between stable equivalence and irreducible maps. Then we have $\underline{g f}=0$, and also $g^{\prime} f^{\prime}=0$. By Lemma 2.1(a) we can then conclude that $\alpha S_{2} \cong T_{2}$.

LEMMA 2.3. Let $\alpha: \underline{\bmod \Lambda} \rightarrow \underline{\bmod \Lambda^{\prime}}$ be a stable equivalence between indecomposable Nakayama algebras, and assume that each indecomposable projective $\Lambda$-module and $\Lambda^{\prime}$-module has length at least 3 . If for some simple $\Lambda$-module $S_{1}, \alpha S_{1}$ is not simple, then the admissible sequences for $\Lambda$ and $\Lambda^{\prime}$ are both constant, and are hence the same.

Proof. Assume that $\alpha S_{1}$ is not simple. $\left(, S_{1}\right)$ denotes the functor from $\bmod \Lambda$ to $A b$ given by $\left(, S_{1}\right)(A)=\operatorname{Hom}\left(\underline{A}, \underline{S_{1}}\right)$. By [9, Proposition 1.1] we know that $\left(, S_{1}\right)$ is uniserial and that $\alpha S_{1} \cong Q_{1} / \mathfrak{r}^{n-1} Q_{1}$, where $Q_{1}$ is an indecomposable projective $\Lambda^{\prime}$-module of length $n \geqslant 3$. We further know that

$$
l\left(P_{1}\right)-1=l\left(\left(, \underline{S_{1}}\right)\right)=l\left(, \underline{Q_{1} / \mathrm{r}^{n-1} Q_{1}}\right)=l\left(Q_{1}\right)-1,
$$

where $P_{1}$ denotes a projective cover for $S_{1}$. The chain $S_{2} \stackrel{f}{\rightarrow} P_{1} / \mathrm{r}^{2} P_{1} \stackrel{g}{\rightarrow} S_{1}$ of irreducible maps gives rise to a chain of irreducible maps $\alpha S_{2} \stackrel{f^{\prime}}{\rightarrow} \alpha\left(P_{1} / \mathrm{r}^{2} P_{1}\right) \stackrel{g^{\prime}}{\rightarrow} \alpha S_{1}$, where $\underline{g f}$ is zero. By Lemma $2.1, \mathrm{r} Q_{1}$ is not projective, and $\alpha S_{2} \cong \mathrm{r} Q_{1}$. Since $\mathrm{r} Q_{1}$ cannot be simple, we have as above that $\mathrm{r} Q_{1} \cong Q_{2} / \mathrm{r}^{m-1} Q_{2}$, where $Q_{2}$ is an indecomposable projective $\Lambda^{\prime}$-module of length $m \geqslant 3$, and also that

$$
l\left(P_{2}\right)-1=l\left(, \underline{S_{2}}\right)=l\left(, \underline{Q_{2} / \mathrm{r}^{m-1} Q_{2}}\right)=l\left(Q_{2}\right)-1 .
$$

Here $P_{2}$ denotes a projective cover for $S_{2}$. Since clearly $l\left(Q_{1}\right)=l\left(Q_{2}\right)$, we have by the above that $l\left(P_{1}\right)=l\left(P_{2}\right)$. The proof of the lemma is then easily finished.

We are now ready to prove our desired result.

THEOREM 2.4. Let $\Lambda$ and $\Lambda^{\prime}$ be stably equivalent indecomposable Nakayama 
algebras where each indecomposable projective module has length at least 3. Then $\Lambda$ and $\Lambda^{\prime}$ have the same admissible sequences.

Proof. Let $\alpha: \bmod \Lambda \rightarrow \bmod \Lambda^{\prime}$ be an equivalence. Assume first that there

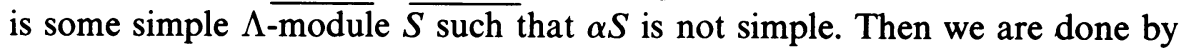
Lemma 2.3. If for every simple $\Lambda$-module $S, \alpha S$ is simple, we are done using Lemma 2.2 since $l(P)-1=l((, \underline{S}))$, where $P$ is a projective cover for $S$ [9]. This finishes the proof of the theorem.

We have only dealt with indecomposable Nakayama algebras. This is no loss of generality because if $\Lambda$ and $\Lambda^{\prime}$ are stably equivalent Nakayama algebras where each indecomposable projective module has length at least 3 , and $\Lambda=\Lambda_{1} \times \cdots \times \Lambda_{n}$ and $\Lambda=\Lambda_{1}^{\prime} \times \cdots \times \Lambda_{m}^{\prime}$ are decompositions into products of indecomposable algebras, then $m=n$, and the numbering can be made such that $\Lambda_{i}$ and $\Lambda_{i}^{\prime}$ are stably equivalent. This is a direct consequence of the following result.

Proposition 2.5. Let $\Lambda$ be a Nakayama algebra such that each indecomposable projective $\Lambda$-module has length at least 3. Then $\Lambda$ is indecomposable if and only if $\bmod \Lambda$ is an indecomposable category.

Proof. Assume that $\Lambda$ is indecomposable, and let $S_{1}, \ldots, S_{n}$ be the simple $\Lambda$-modules, ordered in a way such that $S_{i+1} \cong \mathrm{r} P_{i} / \mathrm{r}^{2} P_{i}, i=1, \ldots, n-1$, $S_{1} \cong \mathrm{r} P_{n} / \mathrm{r}^{2} P_{n}$. We have nonzero morphisms $\underline{S_{i+1}} \rightarrow P_{i} / \mathrm{r}^{2} P_{i}$ and $P_{i} / \mathrm{r}^{2} P_{i} \rightarrow S_{i}$. And if $X$ is an arbitrary indecomposable nonprojective $\Lambda$-module, there is clearly some $S_{i}$ such that $\operatorname{Hom}\left(\underline{X}, \underline{S_{i}}\right) \neq 0$. From this we conclude that $\underline{\bmod \Lambda}$ is indecomposable.

If $\underline{\bmod \Lambda}$ is indecomposable and $\Lambda$ has no semisimple ring summand, then $\Lambda$ is clearly indecomposable.

We end this section by pointing out that if $\Lambda$ and $\Lambda^{\prime}$ are stably equivalent indecomposable Nakayama algebras, then we cannot in general conclude that they have the same admissible sequences. For example, if $k$ is a field, then $k[x] /\left(x^{2}\right)$ and $\left(\begin{array}{ll}k & 0 \\ k & k\end{array}\right)$ are stably equivalent Nakayama algebras with admissible sequences $(2)$ and $(2,1)$ respectively.

\section{REFERENCES}

1. M. Auslander and I. Reiten, Stable equivalence of artin algebras, Proc. Conf. on Orders, Groups Rings and Related Topics, Lecture Notes in Math., vol 353, Springer-Verlag, New York, 1973.

2. __ Stable equivalence of dualizing R-varieties. V, Advances in Math. 17 (1975), $167-195$

3. __ Representation theory of artin algebras. IV: Invariants given by almost split sequences, Comm. Algebra 5 (1977), 443-518.

4. Representation theory of artin algebras. V; Methods for computing almost split sequences and irreducible morphisms, Comm. Algebra 5 (1977), 519-554.

5. K. R. Fuller, Generalized uniserial rings and their Kupisch series, Math. Z. 106 (1968), $248-260$.

6. K. R. Fuller, J. Haack and H. Hullinger, Stable equivalence of uniserial rings, Proc. Amer. Math. Soc. 68 (1978), 153-158.

7. J. A. Green, Walking around the Brauer tree, J. Austral. Math. Soc. 17 (1974), 197-213. 
8. H. Kupisch, Beiträge zur Theorie nichthalbeinfacher Ringe mit Minimalbedingung, Crelles J. 201 (1959), 100-112.

9. I. Reiten, Stable equivalence of dualizing $\boldsymbol{R}$-varieties. VI. Nakayama dualizing $\boldsymbol{R}$-varieties, Advances in Math. 17 (1975), 196-211.

10. 211-212.

11. Almost split sequences for group algebras of finite representation type, Trans. Amer. Math. Soc. 233 (1977), 125-136.

12. __ Algebras stably equivalent to Nakayama algebras of Loewy length at most 4, Austral. Math. Soc. (to appear).

Department of Mathematics, Brandeis University, Waltham, MassaChUSETts 02154

Department of Mathematics, University of Trondhem, NLHT, 7000 Trondhem, NoRWAY (Current address) 